\title{
A Regularist Approach to Mechanistic Type-Level Explanation
}

\author{
Beate Krickel
}

\begin{abstract}
Most defenders of the new mechanistic approach accept ontic constraints for successful scientific explanation (Illari [2013]; Craver [2014]). The minimal claim is that scientific explanations have objective truthmakers, namely mechanisms that exist in the physical world independently of any observer and that cause or constitute the phenomena-to-be-explained. How can this idea be applied to type-level explanations? Many authors at least implicitly assume that in order for mechanisms to be the truthmakers of type-level explanation they need to be regular (Andersen [2012]; Sheredos [2015]). One problem of this assumption is that most mechanisms are (highly) stochastic in the sense that they 'fail more often than they succeed' (Bogen [2005]; Andersen [2012]). How can a mechanism type whose instances are more likely not to produce an instance of a particular phenomenon type be the truthmaker of the explanation of that particular phenomenon type? In this paper, I will give an answer to this question. I will analyze the notion of regularity and I will discuss Andersen's suggestion for how to cope with stochastic mechanisms. I will argue that her suggestion cannot account for all kinds of stochastic mechanisms and does not provide an answer as to why regularity grounds type-level explanation. According to my analysis, a mechanistic type-level explanation is true if and only if at least one of the following two conditions is satisfied: the mechanism brings about the phenomenon more often than any other phenomenon ('Comparative Regularity') or the phenomenon is more often brought about by the mechanism than by any other mechanism/causal sequence ('Comparative Reverse Regularity').
\end{abstract}

\section{Introduction}

2. The Minimal Characterization of Mechanisms and Mechanistic Explanation

3. Regularity

4. Reverse Regularity

5. Comparative Regularity and Reverse Regularity

6. Multiple Realization, Multifunctionality and the Individuation of Types

7. Conclusion

\section{Introduction}

According to the new mechanistic approach, mechanisms are crucial for the special sciences (especially the life sciences). Most prominently, the new mechanists argue that explanation in the special sciences is mechanistic in the sense that phenomena are explained by referring to mechanisms that are responsible for them. An example of such an explanation is the explanation of neurotransmitter release (MDC [2000]; Craver [2007a]). Roughly, the mechanism that explains neurotransmitter release consists of an action potential that reaches the axon terminal that causes calcium channels to open, which causes calcium to influx into the axon terminal, which in the end causes synaptic vesicles filled with neurotransmitters to 
fuse with the membrane of the axon terminal, which leads to a release of the neurotransmitters into the synaptic cleft. Importantly, in this example, the mechanism is not supposed to explain only one particular occurrence of neurotransmitter release. Rather, it is meant to explain how neurotransmitter release is brought about in general. In this sense, mechanistic explanations are usually type-level explanations (but they need not be).

Additionally, most new mechanists hold that mechanistic explanation is ontic in some sense. They differ in how these ontic norms are spelled out. According to the so-called 'ontic view of scientific explanation' (Craver [2007a], [2014]), explanations are 'objective portion[s] of the causal structure of the world' that 'are not true or false', 'they just are' (Craver [2014], p. 40) and do 'not depend on the existence of intentional agents' as, for example, explanatory models do (Craver [2014], p. 36). Furthermore, ontic explanations 'consist in all and only the relevant features of the mechanism in question' (Craver [2014], p. 40). Hence, according to the ontic view of scientific explanations, explanations and mechanisms are one and the same thing that exists in the external world independently of us.

The ontic view has been criticized mainly on the grounds that explanation cannot be successful without obeying some epistemic norms (Bechtel [2008], p. 18; Wright [2012]; Sheredos [2015]). Still, all or at least most new mechanists seem to accept a weakened version of the ontic account. According to the weak reading of the ontic view, good scientific explanations have to account for ontic norms and epistemic norms (Illari [2013]; Craver [2014]). Here, the ontic norms can be interpreted in terms of the truth of an explanation: an explanation has to describe the causal structure of the physical world in a correct way to be true. In this sense, explanations are descriptions or models of the world and mechanisms are their truthmakers. Still, in order to be a good explanation truth might not be sufficientepistemic norms (like, for example, understandability) might have to be satisfied as well. ${ }^{1}$ In this paper, I will presuppose the weak interpretation of the ontic view. Hence, I will take explanations to be descriptions or models that are not identical with mechanisms but are made true by them (for a discussion of type-level explanation/generalizations in the context of the strong reading of the ontic view see Sheredos [2015]).

Presupposing a weak reading of the ontic view, the new mechanists seem to be committed to the view that also in the case of mechanistic type-level explanations the truthmakers are mechanisms. How can mechanisms be the truthmakers of type-level explanations like the explanation of neurotransmitter release described above? One answer to this question might

\footnotetext{
${ }^{1}$ The 'epistemic view' of scientific explanation might be interpreted accordingly: its strong reading is that scientific explanation has to obey purely epistemic norms - there can be good explanations that are not true; the weak reading is identical to the weak reading of the ontic claim.
} 
be found in what I will call the 'regularity-based characterization of mechanisms'. Many mechanists assume that mechanisms are regular in some sense (MDC [2000]; Andersen [2012]). For example, Peter Machamer, Lindley Darden and Carl Craver (MDC [2000]) characterize mechanisms as involving 'regular changes', and as 'regular in that they work always or for the most part in the same way under the same conditions' (MDC [2000], p. 3). Benjamin Barros argues that '[s]ome degree of regularity is necessary to make predictive explanations' ([2008], p. 310). Similarly, Lane DesAutels holds that 'if we do not require mechanisms to be productive of regular or invariant change at all, then [...] mechanisms no longer serve to ground prediction and support intervention strategies' ([2011], p. 921). Finally, Holly Andersen ([2012]) agrees that type-level explanation is crucial in the sciences and argues that this requires mechanisms to be regular:

\begin{abstract}
Mechanistic explanatory practices in the sciences hinge on considering single instances as instances of a type, and on providing explanations based on mechanisms that constitute a type of causal chain, not merely a single instance of one. What happens when a single neuron fires once is not the target of investigation or of explanation. What happens when neurons fire, and why and how it happens, in general: this is what mechanisms can explain, but only if they retain regularity. (Andersen [2012], p. 429)
\end{abstract}

Hence, it could be argued that mechanisms are the truthmakers of type-level explanations because mechanisms are regularly occurring types that bring about the phenomenon to be explained. Although this view seems to provide a promising route to mechanistic type-level explanation, one problem for the regularity-based characterization of mechanisms is, what I will call, the 'Bogen-Argument' (Bogen [2005]; see also Section 3). Roughly, Jim Bogen argues that many mechanisms work rather irregularly. For example, the neurotransmitter release mechanism fails more often that it succeeds (I will call mechanisms that are more likely to fail than to succeed 'high failure mechanisms'). To think that this irregularity is only due to a lack of knowledge and that we can always establish a full-fledged regularity, according to Bogen, is an 'article of faith' (Bogen [2005], p. 400).

The insight that explanatory power does not depend on deterministic generalizations was one motivation for philosophers of science to abandon the classical D-N model of scientific explanation (Salmon [1989], chapters 3 and 4). For example, according to Wesley Salmon's ([1971]) statistical relevance approach an explanation is good if it cites all and only statistically relevant factors (factors that change the probability of the occurrence of an event) and it is irrelevant for the explanation whether these factors render the occurrence of the event-to-be-explained likely or not. According to Peter Railton's ([1978]) 'DeductiveNomological Model of Probabilistic Explanation' the occurrence of an event can be explained even if it was rather unlikely to occur if we can deduce it from a probabilistic law. Both 
approaches accept that in order to explain an event, it need not be deducible from deterministic laws of nature. Furthermore, both philosophers can be considered early new mechanists due to their commitment to the view that explanation consists in 'revealing the mechanisms at work in the world' (Salmon [1989], p. 156). In order to account for this doctrine, Salmon develops a process theory of causation that is supposed to provide the resources for satisfying the ontic constraints on successful (statistical) explanation (Salmon [1984]). Railton holds that revealing 'the mechanisms at work in the world' can be done by showing that the probabilistic law itself can be explained on the basis of the underlying mechanisms. Still, both approaches do not seem to be of much help for present purposes. The reason is that Salmon's as well as Railton's approach are accounts of token-level explanation and it is not clear how exactly they can be extended to cover type-level explanation.

A more promising strategy is to look for a more liberal notion of regularity that still can account for the truth of type-level explanation. Andersen ([2012]), for example, admits that regularity cannot require a mechanism to always bring about the phenomenon at question (Andersen [2012], p. 419). Rather, she accepts that a mechanism can be regular even if it fails to bring about the phenomenon more often than it succeeds. According to Anderson, highfailure mechanisms are regular if either, in cases of failure, one can identify interfering factors, or if there is a consistent percentage of times where the mechanism succeeds (which might be rather low) (Andersen [2012], p. 421).

Although Andersen provides a compelling overview of the ways in which mechanisms might be regular, her account is problematic. First, not all high-failure mechanisms are regular in either of the two senses she defined. Second, she does not provide an explicit account of how the notion of regularity can be implemented into an account of mechanistic type-level explanation. Questions that remain unanswered are: How can we justify that a mechanism M explains a phenomenon $\mathrm{P}$ even if $\mathrm{M}$ does not bring about $\mathrm{P}$ in most cases? Can high failure mechanisms be truth-makers of mechanistic explanations at all? If high-failure mechanisms can be truth-makers of explanations, what distinguishes these explanations from false explanations? Do explanations have to refer to deterministic mechanisms (mechanisms that always bring about the relevant phenomenon) in order to be true? In this paper, I will develop an account of mechanistic type-level explanations that provides answers to these questions. I will show under which conditions high-failure mechanisms can be truthmakers of mechanistic type-level explanations.

The paper proceeds as follows: in Section 2, I will explain how mechanisms are commonly characterized in the new mechanistic literature and how that connects to the issue of scientific type-level explanation. Furthermore, I will present the general idea that some kind of 
regularity is necessary for mechanisms being potential truthmakers of type-level scientific explanation. In Section 3, I will spell out the regularity-based characterization of mechanisms in more detail. I will argue that formulations of regularity-based characterizations that have been developed so far are problematic because they cannot account for mechanisms being truthmakers of type-level explanations. In Section 4, the notion of Reverse Regularity is introduced. I will show how Reverse Regularity can cope with some of the problems classical regularity based views have. But I will argue that this suggestion is not sufficient to account for all cases of mechanistic type-level explanation. In Section 5, I will show that we need comparative notions of regularity in order to provide a satisfying account of mechanistic typelevel explanation. In Section 6, I will discuss multiple realization and multiple functionality as challenges for my approach and argue that meeting these challenges crucially depends on a satisfying account of how to individuate mechanism and phenomenon types. Section 7 concludes.

\section{The Minimal Characterization of Mechanisms and Mechanistic Explanation}

There is an ambiguity in how the term 'mechanism' is used in the new mechanistic literature (Nicholson [2012]). Sometimes the term is used to refer to machine-like structures (for example Glennan [1996]); at other times, it is meant to refer to causal sequences. Here, I am concerned with the second use since it is more central to the new mechanistic thinking. At least what has come to be known as the 'Minimal Characterization' (MC) of mechanisms (Glennan [under review]) — which has become generally accepted amongst the new mechanists - describes a causal sequence rather than a machine-like structure ${ }^{2}$ :

(MC) Mechanisms are entities and activities organized such that they bring about a certain phenomenon.

Entities are taken to be objects like neurons, cells, ions, rats and the like (Craver [2007a], pp. 128-33). Activities are taken to be the things the entities do that allow them to causally interact with each other (Craver [2007a], pp. 133-4). Examples are opening, closing, diffusing, binding and the like. Entities and activities must be organized in a certain way in order to bring about the phenomenon (Craver [2007a], pp. 134-9). Entities are spatially organized: for example, they have specific locations relative to each other and they have specific sizes. Activities are temporally organized: they occur in a specific temporal order;

\footnotetext{
${ }^{2}$ For characterizations of mechanisms in accordance with (MC), see (Bechtel and Richardson [1993]; MDC [2000]; Bechtel and Abrahamsen [2005]; Craver [2007a]; Illari and Williamson [2012]).
} 
they have certain durations, frequencies, and the like. Furthermore, entities and activities are what Craver calls 'actively organized' which means that they causally interact in specific ways (Craver [2007a], p. 136). Besides that, it is usually assumed that mechanisms can bring about phenomena in two different ways (note that in both cases, the mechanism is a causal sequence as characterized by (MC)). In etiological mechanistic explanations one refers to mechanisms that cause the phenomenon (for example, the explanation of neurotransmitter release). Constitutive mechanistic explanations refer to mechanisms that underlie or constitute the phenomenon (a common example is the action potential mechanism) (Craver [2007a], chapter 4.8; Kaiser and Krickel [2016]). (MC) characterizes mechanisms as causal sequences rather than machine-like structures because it requires mechanisms to involve various entities that actually interact. A machine is just one object, not many, and it need not be active in order to be a machine. In the following, when I speak of mechanisms as causal sequences I refer to mechanisms as characterized by $(\mathrm{MC})$.

To illustrate (MC), consider the mechanism for neurotransmitter release that was already mentioned in the introduction (Figure 1).

\section{[Insert Figure 1 here]}

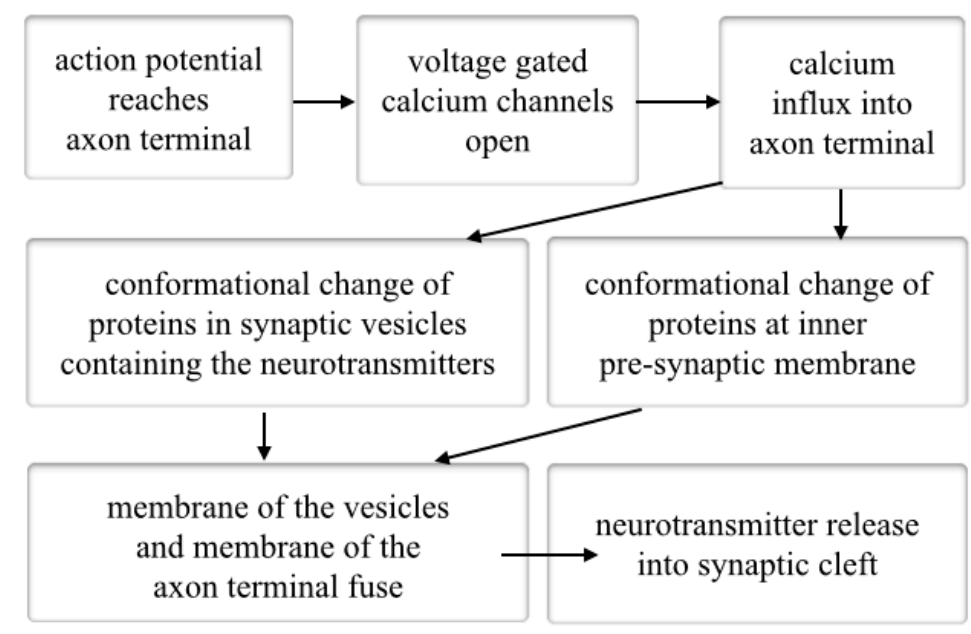

(MC) nicely captures many features of this example: the neurotransmitter release mechanism consists of various entities, such as the axon terminal, ion channels, calcium, proteins, synaptic vesicles, neurotransmitters and so on; it involves activities such as reaching, opening, changing, interacting, fusing; and these entities and activities are organized in various ways. For example, the ion channels have to be at certain locations at the axon terminal membrane; the channels have to open and close in a certain temporal order; and there have to be the right interactions in order for the ion channels to open and for the ions to influx into the axon 
terminal. Furthermore, the neurotransmitter release mechanism brings about neurotransmitter release by causing it.

How does (MC) relate to scientific explanation? As argued in the introduction, mechanisms are supposed to be the truthmakers of scientific explanations. Hence, one might think of (MC) as describing what the truthmaker of a mechanistic explanation look like. What exactly this claim amounts to has to be evaluated in the light of the general mechanistic ontology. The mechanistic ontology seems to be a singularist one. This is suggested by the general acceptance of so-called 'entity-activity dualism' (MDC [2000]; Machamer [2004]; Illari and Williamson [2011]). It is argued that the mechanistic ontology contains (only?) entities (objects) and activities (processes). Both are concrete things that occupy definite space-time regions. Furthermore, Sheredos ([2015]) convincingly argues that the new mechanists cannot accept the existence of Platonic universals due to their naturalism and they cannot accept Aristotelian universals (at least not as truth-makers of type-level generalizations) since the individuation of these universals would presuppose the very generalization they are supposed to make true. ${ }^{3}$ Plausibly, in the mechanistic ontology, types can only exist in so far as tokens exist that can be subsumed under a type description (due to, for example, relevant similarities they share; see Section 6). Hence, mechanistic type-level explanations could be understood as general descriptions, while mechanisms themselves are concrete tokens located in space and time. ${ }^{4}$ For example, Stuart Glennan ([2011]) argues:

[o]ntologically, the crucial point to observe is that mechanisms are not universals but particulars. They are structured collections of parts which occupy a certain region of space and which interact over a certain definite period of time. We characterize these mechanisms by generalizations because very often a mechanism's behavior is repeatable. (Glennan [2011], p. 810)

\footnotetext{
${ }^{3}$ Sheredos ([2015]) discusses different suggestion of how types might be integrated into the mechanistic ontology. He argues that the most plausible approach to universals that is compatible with the new mechanistic view is Aristotelian Realism, according to which universals can exist in multiple places and times by being colocated in each of its instances. Based on his discussion of the ontology of types, Sheredos develops an argument against the ontic view of explanation: the acceptance of Aristotelian types is the consequence of a prior acceptance of epistemic norms of generality that cannot be inferred from the ontic view. As already noted, I am not committed to the ontic view of explanation here. Rather, I do accept that type-level explanations are descriptions that might have been developed on the basis of several epistemic norms. I am only committed to the view that the truth of an explanation depends on the mapping between the descriptions and certain features of the tokens that fall into the scope of the description. Furthermore, I am not committed to Aristotelian realism with regard to types either. My arguments seem to be compatible with nominalism as well.

${ }^{4}$ In this paper, I do not address the question of whether mechanisms themselves are types or concrete particulars. Maybe a token causal chain of organized entities and activities is a mechanism only if it falls under a general mechanistic description (defenders of the regularity-based characterization of mechanisms seem to think that; see introduction and below). Maybe causal chains can be mechanisms even if they do not fall under a general mechanistic description (Glennan argues for this claim; see Glennan [2010]). I do not want to settle this issue here.
} 
The commitment to ontic constraints on mechanistic explanation plus the endorsement of a singularist ontology has important consequences for type-level explanations: type-level claims about mechanisms, phenomena and their relation have to have singularist truthmakers. The claim that the neurotransmitter release mechanism explains neurotransmitter release, for example, is somehow made true by features of the token instances of the mechanism and the phenomenon and the causal relations in which they stand.

In this paper, I will assume that mechanism types explain because their instances cause or constitute (instances of) phenomena. In other words, I will presuppose a singularist interpretation of causation and constitution (according to which the relata of causation and constitution are primarily tokens). This assumption seems to be justified given the singularist ontological commitments of the new mechanists. ${ }^{5}$ Of course, a singularist ontology is compatible with a generalist view (the view that the relata of causation and constitution are primarily types) as well if the truthmakers of the generalist causal/constitutive claims are taken to be singularist. I opt for a singularist view of causation and constitution because it allows me to ignore the question of what causation and constitution exactly are for a moment. I will assume that we have a more or less good understanding of what it means to say that a mechanism token causes/constitutes a phenomenon token (see Kaiser and Krickel [2016]) for an analysis of constitution between tokens) and concentrate on how we make sense of typelevel explanation given knowledge about token-level causation/constitution. But my considerations will not depend on the acceptance of singularist view of causation and/or constitution. ${ }^{6}$

Based on these assumptions, a first attempt to spell out an account of type-level explanation might be the following: a mechanism type explains a phenomenon type if and only if every instance of the former causes or constitutes ${ }^{7}$ an instance of the latter (a similar

\footnotetext{
${ }^{5}$ Among the new mechanists, singularist as well as generalist views with regard to causation and constitution can be found: mechanists endorsing activity-causation are singularists (Machamer [2004]; Glennan [2011]); those that defend interventionist theories of causation or constitution are generalist (see Craver [2007b]; Baumgartner and Gebharter [2015]; Romero [2015]; for a generalist view of constitution not in terms of interventionism see Harbecke [2010] and Couch [2011]).

${ }^{6}$ Everything I am going to say can be reformulated in terms of generalist views of causation and constitution. Then, the basic claim made above 'mechanism types explain because their instances cause/constitute phenomena' has to be reformulated: 'mechanism types explain because they cause or constitute phenomena'the question, then, would be how to spell out type-level causation/constitution such that the singularist truthmakers of the generalist causal/constitutive claims are at the same time plausible truth-makers of type-level mechanistic explanation (given the problems I am going to address in what follows).

${ }^{7}$ Most of the debate on regularity in the context of the new mechanistic debate seems to be framed in terms of etiological mechanistic explanation (the neurotransmitter release mechanism is a common example when regularity is discussed which is a mechanism that causes its phenomenon). How the considerations concerning regularity apply to constitutive mechanistic explanation is a matter of debate. Some authors argue that mechanistic constitution is a necessitation relation (Harbecke [2010]; Couch [2011]). Others argue that mechanisms have to be distinguished from background factors, where the latter are necessary for the
} 
idea regarding the relation between type- and token-level causal claims can be found in Hausman [2005], p. 38). Figure 2 illustrates this idea.

[Insert Figure 2 here]

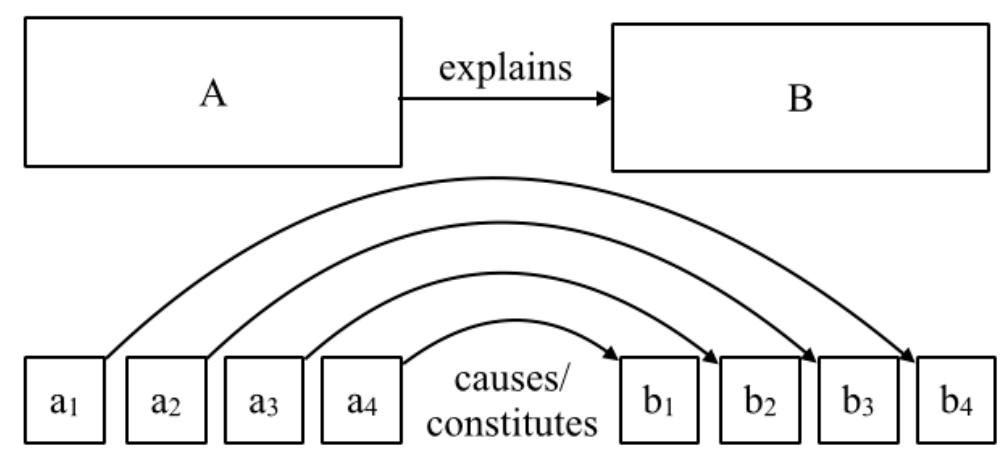

In Figure 2 the capital letters A and B stand for types and the small letters stand for the corresponding tokens/instances. Applied to the case of mechanisms, A stands for a mechanism type, whereas B stands for a phenomenon type. The mechanism type A explains phenomenon type B if and only if every instance of the mechanism A causes/constitutes an instance of phenomenon B.

The idea that type-level mechanistic explanation depends on some kind of constant causal or constitutive conjunction between instances of the mechanism and instances of the phenomenon is reflected by what I have called the 'regularity-based characterization' of mechanisms. In the following section, I will spell out the regularity-based characterization of mechanisms in more detail.

\section{Regularity}

Defenders of regularity-based characterizations (MDC [2000]; Barros [2008]; Andersen [2012]) usually argue that a causal sequence, in order to deserve the label 'mechanism', has to be regular. In other words, these authors deny that one-off causal chains can be mechanisms. Stuart Glennan ([2010]), in contrast to that, argues that there can be historical mechanisms which he calls 'ephemeral mechanisms' that occur only once. I do not want to settle this issue here. Still, the question of how the notion of regularity should be understood in the context of the new mechanistic approach seems to arise for both camps. Everyone who accepts that there

phenomenon to occur but are not part of the mechanism (Craver [2007a], chapter 4.8). The latter view implies that the mechanism is not sufficient for the phenomenon, and thus might fail to constitute the phenomenon. Plausibly, if one accepts the Bogen-argument in the context of etiological mechanistic explanation, one has good reasons to accept it for constitutive mechanistic explanation as well. Hence, mechanistic constitution might be probabilistic in some sense as well. 
can be true type-level mechanistic explanations has to tell a story of how mechanisms can be the truthmakers of these explanations; and the most plausible answer to that question seems to be that (at least some) mechanisms are regular in some sense.

How do we have to understand regularity such that this notion can be used to make sense of mechanistic type-level explanation? In order to answer this question we should clarify under which conditions a possible answer to this question is good, and when it is not. In what follows, I will assume the two following criteria of adequacy: first, an approach to regularity as the basis for true mechanistic explanations has to account for clear cases of true mechanistic explanation. Second, it has to make intelligible how or why regularity grounds type-level mechanistic explanation. That means, it should clarify why a mechanism explains a phenomenon rather than another and it should provide a plausible story as to why, on the grounds of the respective notion of regularity, one explanation qualifies for being true while another does not.

Furthermore, before developing an approach to regularity, we have to clarify what exactly the bearer of regularity is supposed to be. Plausibly, regularity is a feature of sequence types. In the context of an analysis of mechanistic type-level explanation, the sequence type that has to be regular consists of the mechanism type and the phenomenon type. ${ }^{8}$ (In case of etiological explanations we are dealing with 'causal sequences'; in constitutive explanations we are dealing with 'constitutive pairs' where the mechanism and the phenomenon occur at the same time - for the sake of simplicity I will speak of 'sequences' in both cases). In what follows, I will often speak of a causal/constitutive sequence type that consists of two sequence types $\mathrm{A}$ and $\mathrm{B}$. This is meant to be an abbreviation of the phrase that there is a (sequence) type A whose instances cause/constitute instances of another (sequence) type B (or, if one endorses a generalist view of causation/constitution, one might say that A causes/constitutes B).

Now, what does it mean to say that the sequence consisting of the mechanism and the phenomenon is regular? Andersen ([2012]) holds that regularity in this context is a factual notion rather than a counterfactual one. She argues that ' $[\mathrm{t}]$ he notion of regularity $[\ldots]$ is actual and not counterfactual, namely, multiple occurrences in the actual world' (Andersen

\footnotetext{
${ }^{8}$ Andersen ([2012]) discusses different causal sequences as bearers of regularity that are crucial for the question of how regular a mechanism is. She argues that in order to determine the overall regularity of a mechanism, one has to determine how regularly the relevant inputs of a certain mechanism occur, how reliably the mechanism is triggered by a certain input, how stable the connections between the mechanism's components are, and how reliably the mechanism brings about the phenomenon. Here, we can ignore the former and focus on the latter sequence because, for present purposes, it is not relevant whether, for example, the mechanism occurs regularly given the input. If one is interested in mechanisms as, for example, supporting interventionist strategies, one might have to talk about the regularity of other causal sequences as well such as the input-mechanism sequence or the sequence consisting of the mechanism's components.
} 
[2012], p. 430). The notion of regularity that Andersen seems to have in mind can be defined as follows:

(Factual Regularity) A causal/constitutive sequence type is factually regular iff it has multiple ${ }^{9}$ actual instances.

The claim that mechanisms are regular in this sense implies that the sequence consisting of the mechanism type and the phenomenon type has to have multiple instances. In other words, mechanisms correspond to types of sequences that have multiple instances in the actual world that successfully bring about (cause or constitute) instances of a particular phenomenon type.

The assumption that mechanisms are factually regular in the sense just presented is plausible at least when restricting the analysis to biological mechanisms. First, clear cases of mechanisms in the life sciences that are central examples in the new mechanistic debate are indeed factually regular (like the neurotransmitter release mechanism, the action potential mechanism, the spatial memory mechanism). Second, biological mechanisms develop in the course of biological evolution. Natural selection, which is the motor of biological evolution, results in individuals of the same species being made up in the same way such that they (or their parts) are disposed to give rise to the same mechanisms. Third, as a matter of fact it is rather difficult to find valid examples of biological causal sequences that are not factually but merely counterfactually regular (for which it is true that they occurred only once and if certain circumstances had obtained again, a causal sequence of the same type would have occurred again). Fourth, even if biological mechanisms might be counterfactually regular, the idea is that this modal knowledge is not relevant for the causal and explanatory power of a particular mechanism (one reason for that is the notorious difficulty to spell out a semantics for counterfactuals; Bogen [2004], [2005]).

So far, our characterization of regularity fits well to our considerations made in Section 2 (see Figure 2): a mechanism type A explains a phenomenon type B if and only if sequence type consisting of $\mathrm{A}$ and $\mathrm{B}$ has multiple instances. Unfortunately, this requirement has a reading that is too strong with regard to the present purpose. It is too strong if we interpret Factual Regularity as a deterministic notion:

\footnotetext{
${ }^{9}$ What exactly does it mean to hold that a type has 'multiple instances'? For present purposes, it suffices to assume that 'multiple instances' means to have more than one instance (the type is not a singular occurrence and it is not merely potentially regular).
} 
(Deterministic Regularity) A causal/constitutive sequence type consisting of A and B is deterministically regular iff all instances of A cause/constitute instances of B.

Applying Deterministic Regularity to mechanistic type-level explanations amounts to the claim all instances of a mechanism (A) have to bring about an instance of the phenomenon (B) in order for the mechanism to explain the phenomenon. This requirement is problematic because it is commonly accepted that mechanisms can fail to bring about the phenomenon they are supposed to explain. The idea that mechanisms can fail makes sense only if we interpret it as a claim about types that have instances that do not bring about the phenomenon that is individuative of the mechanism type. For example, in order to make sense of the claim that the neurotransmitter release mechanism has a certain failure rate, we have to assume that there are causal sequences that belong to the mechanism type 'neurotransmitter release mechanism' although they do not produce neurotransmitter release and even though they might not involve all the causal steps that are characteristic of the neurotransmitter release mechanism. In order to be able to determine when a certain mechanism belongs to a certain type or not a more careful analysis of the identity criteria of mechanism types and tokens is required. I will make some remarks on that issue in Section 6.

The idea that there are stochastic mechanisms - mechanism types that have instances that do not bring about the phenomenon - is commonly accepted among the new mechanists (Bogen [2005]; Barros [2008]; DesAutels [2011]; Andersen [2012]). Figure 3 illustrates the idea of stochastic mechanisms.

[Insert Figure 3 here]

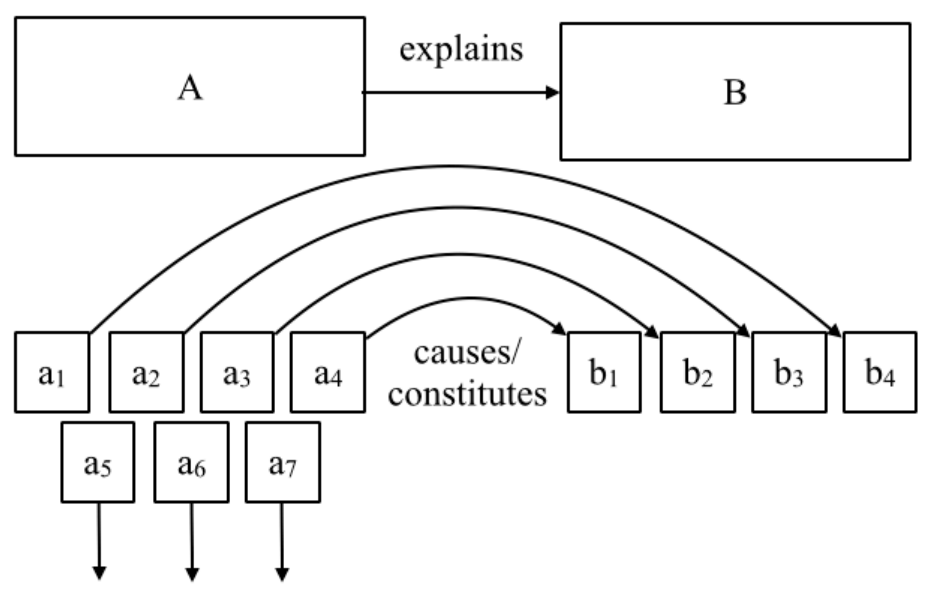

In Figure 3, the stochastic nature of the relationship between a mechanism and a phenomenon is represented. The arrows pointing down indicate that the instances of $\mathrm{A}$ do not 
cause/constitute an instance of B. By putting it this way, I am not claiming that the stochastic nature of mechanisms should be analyzed in terms of a frequentist account of probability. In this paper, I do not discuss which interpretation of probability is most adequate in the context of stochastic mechanisms (for arguments against a frequentist interpretation of stochastic mechanisms and in favor of propensities see DesAutels [2015]); for a mechanistic interpretation of probability see Abrams [2012]).

How can stochastic mechanisms be the truthmakers of mechanistic type-level explanations? One straightforward answer might be to say that a mechanism type explains a phenomenon type if the majority of instances of the mechanism type brings about the phenomenon. This strategy relies on a modified notion of regularity:

(Stochastic Regularity)
A causal/constitutive sequence type consisting of type A and type $\mathrm{B}$ is stochastically regular iff more than $50 \%$ of instances of A cause/constitute instances of B.

One might argue that a value $>50 \%$ is sufficient for a causal sequence to count as regular since it implies that there are more instances of a mechanism that do cause/constitute the phenomenon than instances that do not cause/constitute the phenomenon. Still, this suggestion is problematic for two reasons: first, it fails to account for the second criterion of adequacy. It does not provide an answer to the question of why, for example, a mechanism whose instances bring about the phenomenon only in $50 \%$ of the cases cannot be explanatory. Why should the corresponding explanation be false while an explanation referring to an only slightly more regular mechanism should be true? Drawing a demarcation line in this way seems to be rather arbitrary. Neither abandoning the demand for a value $>50 \%$ and simply requiring a 'high' value does help since it is either unclear what 'high' is supposed to mean or it simply leaves undetermined a yet to be defined value. Second, the suggestion fails because it is commonly accepted that there are mechanisms that fail more often than they succeed (Bogen [2005], p. 400; see also Barros [2008]; DesAutels [2011]; Andersen [2012]). Figure 4 illustrates the idea of a high-failure mechanism (there are more as that do not bring about instances of $\mathrm{B}$ than $a$ s that bring about instances of $\mathrm{B}$ ).

[Insert Figure 4 here] 


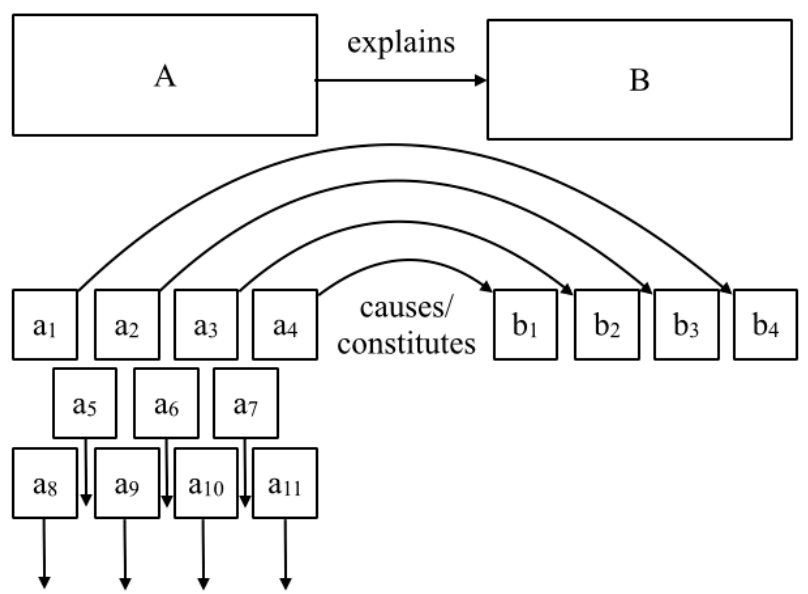

High failure mechanisms are regular in the factual sense-they have multiple instances. But they are not stochastically regular: more instances of the particular mechanism type fail to bring about the phenomenon than succeed in bringing about the phenomenon. The neurotransmitter release mechanism is taken to be such a high failure mechanism (Bogen [2005]; Andersen [2012]). Still, the neurotransmitter release mechanism is considered a true type-level explanation of neurotransmitter release.

Another example of a high-failure mechanism is the cancer mechanism. Consider Figure 5 as an illustration of the cancer mechanism.

[Insert Figure 5 here]

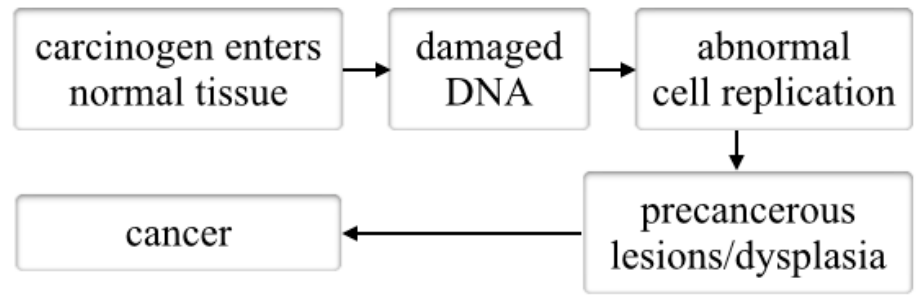

When a carcinogen enters healthy tissue this leads to damages of the DNA of the particular cell. The cell replicates which leads to the proliferation of daughter cells that inherit the damaged DNA. This leads to abnormal cell replication. The result is the occurrence of dysplasia and, in the end, cancer. This schematic illustration of the cancer mechanism depicts what is considered a valid type-level explanation of cancer. Still, this mechanism is highly irregular in that in most cases it does not lead to cancer. In their discussion of 'why don't we get more cancer' Mina Bissell and William Hines ([2011]) argue:

[f]rom the moment of conception and throughout life, these cells [cells of the human body] are assailed with radiation, oxidative damage and more. Individuals' own genetic susceptibility, damage from cigarette smoke and pollution, lack of exercise, obesity and, of course, aging itself can cause many oncogenes to get activated and many tumor suppressors to be inactivated. Yet these mutated cells that, 
according to current dogmas, should lose control and become autonomous do not seem to form as many cancers as would be expected from the number of harmful mutations. In fact, the majority of people live cancer-free lives for decades. (Bissell and Hines [2011], p. 320)

Why are scientists justified in saying that the cancer mechanism explains cancer even though, in most cases, it does not lead to cancer? Why can we say that the neurotransmitter release mechanism explains neurotransmitter release even though most instances of the mechanism do not bring about neurotransmitter release?

One strategy might be to argue that high-failure mechanisms ground type-level explanations because they are incomplete descriptions of processes that are in fact deterministic (that means one sticks to Deterministic Regularity). In other words, high-failure mechanism (and stochastic mechanisms in general) do not really exist-if our knowledge about the world were complete, we could describe, for example, the neurotransmitter release mechanism in such a way that all of its instances turn out to be successful. This strategy is problematic. The first reason is what I call the 'Bogen Argument' (a similar argument can be found in Cartwright [1983], p. 49). Bogen presents this argument in his [2005] paper which started the discussion about how regular mechanisms have to be (Bogen seems to presuppose a generalist view of causation; therefore the argument has to be slightly modified to make it applicable to my considerations that presuppose a singularist view of causation; I added expressions in square brackets to indicate the necessary modifications).

\begin{abstract}
Regularists may insist that no matter how unreliable a mechanism seems to be it can't produce [explain] effects unless its operation instances natural regularities. Maybe we don't know how to describe them to a satisfactory approximation. Maybe we don't even know what they are. But all the same, there must be regularities in there somewhere, and the mechanism must operate in accordance with them. That's an article of faith. It doesn't have enough empirical support to rule out the possibility that some causes [mechanisms] operate indeterministicaly and irregularly. As long as there is a non-negligible chance that some causes [mechanisms] operate irregularly, philosophical accounts of causality [mechanistic explanation] should leave room for them. (Bogen [2005], p. 400)
\end{abstract}

The assumption that all apparent cases of irregularity are due to lack of knowledge, according to this argument, is 'an article of faith'. It rests on the assumption that we live in a deterministic world. But we do not know whether the world is like that. Our analysis of mechanisms and type-level mechanistic explanation should better be independent of this assumption.

A second problem for this strategy is that scientists accept high-failure mechanisms as true explanations independently of whether they think that there is more to know that would render the relation deterministic (a similar argument can be found in Cartwright [1983], p. 52). Scientists take the neurotransmitter release mechanism to explain neurotransmitter release even though they do not know what explains its failures. They seem to provide mechanistic explanations of phenomena independently of whether they think that there could 
be a more detailed description of the mechanism that would render the relation between the mechanism and the phenomenon deterministic or not (although it might be an ideal that drives scientific research).

Andersen ([2012]) accepts that most mechanisms are not deterministic and that some even might have high failure rates. According to her, mechanisms are regular enough in order to ground type-level explanation if one of two conditions is satisfied (note that Andersen does not explicitly address the question of how mechanistic type-level explanation works; rather she argues that mechanisms have to be regular in order to count as mechanisms in the first place; Andersen [2012], p. 421). Let us call the first condition 'Frequented Regularity' and the second 'Interrupted Regularity'.

(Frequented regularity) A causal/constitutive sequence type consisting of type A and type $\mathrm{B}$ is frequented regular iff there is a consistent percentage of times where instances of A cause/constitute instances of B.

(Interrupted regularity) A causal/constitutive sequence type consisting of type A and type $\mathrm{B}$ is interruptedly regular iff every time when an instance of $\mathrm{A}$ does not cause/constitute and instance of B interfering factors can be identified.

I agree that Interrupted Regularity provides a notion of regularity that grounds type-level explanation. It states that there is a mechanism that always brings about a phenomenon ceteris paribus (except for cases in which certain interfering factors occur). The cp-clause does not trivialize the assumption of a deterministic generalization because Andersen also requires that we know which factors were responsible for the failure of the mechanism. Still, applying Interrupted Regularity to mechanistic type-level explanation is problematic. First, as a matter of fact, in most cases scientists do not know which factors were interfering with the working of a mechanism. And this does not hinder scientists from accepting the mechanism at issue as a true explanation. Scientists accept the neurotransmitter release mechanism (Figure 1) or the cancer mechanism (Figure 5) as true type-level mechanistic explanations although they do not know exactly which factors lead to failures of these mechanisms. The explanatory status of these mechanisms seems to be independent of whether scientists know the failing conditions or not. Second, the Bogen Argument applies here as well. Interrupted Regularity relies on the idea that the world in principal behaves deterministically. Every time a mechanism fails there is some going-on in the world that is responsible for it. But some mechanisms might be 
inherently stochastic. We should allow for stochastic mechanisms grounding type-level explanations independently of whether there might be a factor that explains why these mechanisms fail if they fail.

Frequented regularity does not require that the world behave in principle deterministically in order for a sequence to be regular. Rather, applied to mechanisms, it merely requires that stochastic mechanisms succeed with a constant frequency. Even if the majority of instances of a particular mechanism type do not bring about the phenomenon, the mechanism counts as regular if, say, every tenth instance does not fail. Andersen ([2012]) argues that high failure mechanisms, such as the neurotransmitter release mechanism, can be said to be regular if regularity is understood in terms of Frequented Regularity. Still, this notion of regularity is problematic. First, the success probabilities of high failure mechanisms need not be consistent. Investigating the neurotransmitter release mechanism, Tiago Branco and Kevin Staras ([2009]) argue that

evidence has accumulated which shows that single terminals contributing to a connection can have release probabilities that are diverse and that can change over time. (Branco and Staras [2009], p. 373)

Second, Frequented Regularity is a non-starter if one does not introduce a minimal value for how often the mechanism has to succeed in order to count as regular. Otherwise, causal chains that have a consistent success rate of $0 \%$ will come out as regular. Integrating Frequented Regularity into an account of type-level explanation, then, would have the odd consequence that a phenomenon is explained by everything that never causes/constitutes it. Hence, we have to determine a minimal value $>0$. Unfortunately, the problem mentioned above now reoccurs: postulating a minimal value seems to be rather arbitrary and leaves open as to why this value is crucial for grounding type-level explanation.

Third, even if a high failure mechanism shows a constant but low success rate, one might still wonder how such a mechanism can ground type-level explanations. Again, this suggestion seems to fail to account for the second criterion of adequacy. If a mechanism brings about a phenomenon in, say, $5 \%$ of cases where it occurs - how can we justify that this mechanism explains this particular phenomenon rather than whatever else it produces in the remaining $95 \%$ of cases? Furthermore, if we assume that explanations referring to highfailure mechanisms can indeed be true explanations, how can we distinguish true explanations from false ones?

A problem similar to the one just raised already brought the traditional D-N-model into trouble. Deductivists were committed to the claim that one can explain a phenomenon only if one can deduce it from a set of laws and initial conditions. In order to cope with stochastic 
events, Hempel ([1965]) developed his inductive statistical account of explanation, according to which stochastic events can be explained only if the explanans confers high probability to the event's occurrence. Hence, deductivists seem to be committed to the claim that one cannot explain stochastic events whose occurrence is rather unlikely.

In the following section, I will discuss a strategy of how to cope with unlikely events (types) that rejects the idea that there can be only explanations of probable events. This strategy is based on a different kind of regularity, which I will call 'Reverse Regularity'.

\section{Reverse Regularity}

One example of Reverse Regularity, in the sense that I want to put forward in what follows, is the one discussed by Scriven ([1959]) and Salmon ([1998]): sometimes having syphilis leads to paresis. But the relation between paresis and syphilis is not deterministic since most people who have syphilis do not have paresis. Still, we want to say that having syphilis causally explains a person's having paresis. According to the present suggestion, the syphilis explains paresis because their relation is reversely regular: all people suffering from paresis also have syphilis. ${ }^{10}$ Dealing with token-level explanation, Salmon gave a similar answer arguing that an explanation need not state necessary conditions in order to be explanatory: ${ }^{11}$

untreated latent syphilis sometimes causes paresis and sometimes does not; but in all cases in which paresis occurs, it is caused by latent untreated syphilis, and latent untreated syphilis explains the paresis. (Salmon [1998], pp. 147-8.)

The core idea of the present suggestion is that a mechanism grounds the explanation of a particular phenomenon type if the mechanism-phenomenon sequence instantiates what I call 'Reverse Regularity'. A preliminary definition of Reverse Regularity is the following:

(Reverse Regularity) A causal/constitutive sequence type consisting of type A and type $B$ is reversely regular iff all instances of $B$ are caused/constituted by instances of $\mathrm{A}$.

\footnotetext{
${ }^{10}$ Thanks to an anonymous reviewer for mentioning this similarity and for suggesting this example.

${ }^{11}$ According to Salmon's 'Statistical Relevance Model' ([1998]), to explain an event is to describe all and only those factors that are statistically relevant to the occurrence of the event. Unfortunately, it is not clear how to apply this account to type-level explanation. The reason is that it is not clear how to determine the reference class that is relevant to the explanation of an event type. Furthermore, as Salmon himself admits, explanation in terms of statistical relevance alone is not sufficient - one has to identify the causal mechanisms as well. The latter is exactly what the new mechanistic approach aims at. The development of an account of the latter with regard to type-level explanation is the aim of this paper.
} 
(Note that as in the case of regularity that was discussed in the previous section, it is plausible to assume that Reverse Regularity is a factual notion). When applied to explanation, this notion of regularity delivers the following account: A explains B if every instance of B is brought about by an instance of $\mathrm{A}$. This does not require that all instances of $\mathrm{A}$ bring about an instance of B in order for A to explain B. Figure 6 illustrates this idea.

[Insert Figure 6 here]

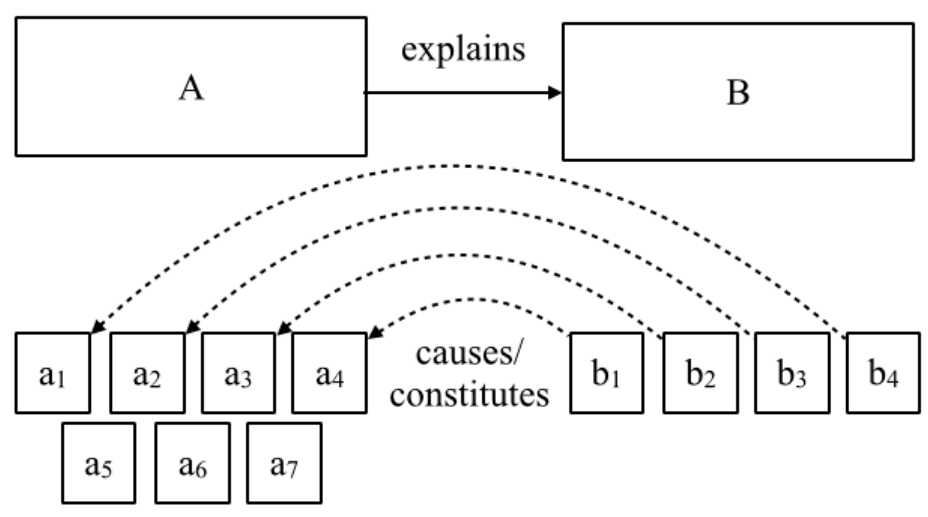

With Reverse Regularity at hand, we can account for high failure mechanisms as grounding true explanations of phenomenon types. Even though a high failure mechanism does not bring about the phenomenon in most of the cases of its occurrence, it can still ground type-level explanation if all instances of the phenomenon are caused by that mechanism. Consider the neurotransmitter release mechanism. Neurotransmitter release is explained by the neurotransmitter release mechanism because all instances of neurotransmitter release are due to the neurotransmitter release mechanism (one indicator that this is the case is the fact that biology textbooks only mention the mechanism for neurotransmitter release depicted above as a mechanism for neurotransmitter release). Similarly, the cancer mechanism depicted in Figure 5 is a true type-level explanation of cancer because in cases where cancer occurs, the mechanism has occurred before - there is no cancer without abnormal cell replication, and there is no abnormal cell replication without DNA damage.

The notion of Reverse Regularity is not only helpful for an analysis of type-level mechanistic explanation. In general, the notion of Reverse Regularity accounts for the fact that scientists often retrodict causes based on their knowledge about mechanisms. For example, physicians infer the causes of symptoms they observe in their patients on the basis of their knowledge about Reverse Regularity relationships between the symptoms and mechanisms that might be responsible for them. In doing so they can evaluate which treatment is most likely to have positive effects. Similarly, knowledge about mechanisms that 
instantiate Reverse Regularity can be used to ground inferences to the best explanation. ${ }^{12}$ Given that we observe a certain phenomenon and we know about different Reverse Regularity relations that phenomenon is known to stand in we are justified in retrodicting that the phenomenon was caused by the event with the highest Reverse Regularity value. Furthermore, knowledge about Reverse Regularity relationships plays a role in mechanism discovery. If scientists are searching for the mechanism of a particular phenomenon, they use their knowledge about Reverse Regularity relationships between the phenomenon and different possible causes the phenomenon has in other contexts where it occurs.

Unfortunately, there is an obvious objection against Reverse Regularity that is analogous to the objection against Deterministic Regularity as discussed in the previous section: most biological phenomena can be brought about in various different ways. Figure 7 illustrates this problem.

[Insert Figure 7 here]

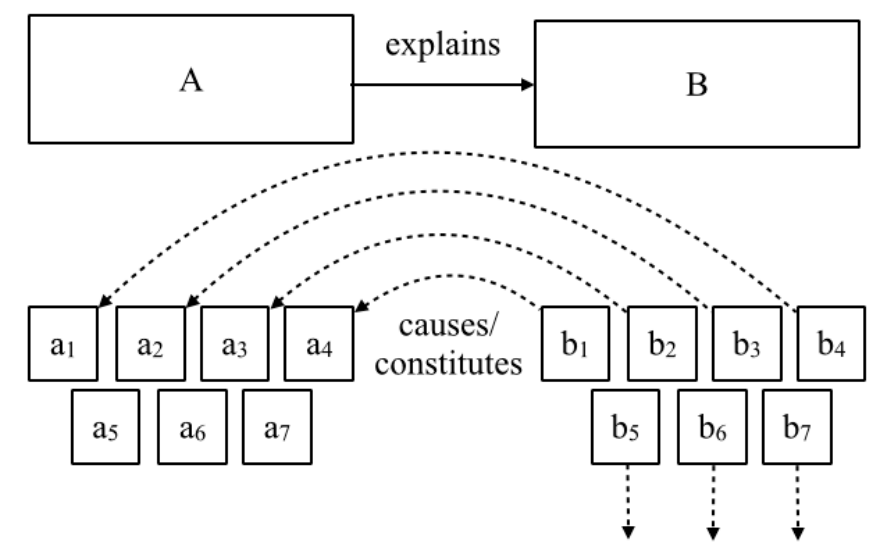

A phenomenon might be brought about by various different causes/constituents and, hence, these phenomena cannot establish Reverse Regularity (in the sense defined above) with regard to any of their causes/constituents. For example, neurotransmitter release might be due to a scientist's manipulation, lighting strikes, or other accidental causes. Can we fix Reverse Regularity such that it accounts for these cases as well? Obviously, we might try strategies analogous to those that were discussed in the previous section in order to account for stochastic mechanisms. We could reformulate Reverse Regularity such that it requires only that most instances of the phenomenon are brought about by the mechanism. But this strategy must fail if there is a reverse analogue to high failure mechanisms - if there are phenomena that can be due to a multitude of different causes/constituents. This latter case might be

\footnotetext{
${ }^{12}$ Thanks to Marshall Abrams for bringing up this idea.
} 
realized in two different ways. First, there might be phenomena that can be due to various different mechanism types. Many diseases and disease-symptoms can be due to many different mechanisms. For example, there are various different mechanisms leading to dizziness. These mechanisms include inadequate blood supply to the brain due to a sudden fall in blood pressure, heart problems or artery blockages, loss or distortion of vision or visual cues, disorders of the inner ear, distortion of brain function by medications such as anticonvulsants and sedatives (Tucci [2013]), or it might be a side effect of certain medical drugs or of consuming too much alcohol. Another example is body temperature homeostasis that is achieved by different mechanisms such as sweating, shivering, and the raising of skin hair. An example taken from cell biology are the different mechanisms that are responsible for the formation of new lumens (i.e. tubular structures) (Sigurbjörnsdóttir et al. [2014]). In none of these cases it is true that most instances of the phenomenon are brought about by one particular mechanism (in Section 6, I will argue that these examples are cases of multiple realization.)

Second, there might be a multitude of different types of singular causes/constituents or one-off causal chains (or 'ephemeral mechanisms', see Glennan [2010]) leading to a given phenomenon where these singular causes together might even be more likely to bring about the phenomenon than the mechanism. Admittedly, it is not easy to find a real biological example for such a scenario. This might be due to the fact that biologists are usually not interested in singular causes of a phenomenon (if there is a mechanism for that particular phenomenon), and therefore do not talk about them in their research papers; or it might be due to the fact that there is no such example. In this paper, I will accept this scenario as possibleit might be the case that a particular phenomenon is more often brought by an instance of a singular cause/one-off causal chain than by a mechanism.

Again, we might reformulate Reverse Regularity in order to account for these cases. We might accept that there are cases where a phenomenon is only rarely brought about by one particular mechanism and still count as regular. We could just determine a rather low minimal value for how many instances of a phenomenon $\mathrm{P}$ have to be brought about by instances of a particular mechanism $\mathrm{M}$ in order for $\mathrm{M}$ to explain $\mathrm{P}$. But, again, the strategy fails for reasons already addressed in the previous section: first, any stipulation of a minimal value must be arbitrary. Second, this strategy leaves open as to why the corresponding notion of Reverse Regularity grounds the truth of type-level explanations.

In the following section, I will make a suggestion of how to solve these problems that relies on a modification of the notions of Reverse Regularity and regularity. 


\section{Comparative Regularity and Reverse Regularity}

So far, all suggestions for how to spell out regularity in the context of mechanistic type-level explanations failed for one or more of the following four reasons:

1) The suggestion relied on an arbitrarily chosen minimal value that determines which sequences count as regular and which do not.

2) The suggestion could not make sense of why a particular notion of regularity grounds the truth of a type-level explanation.

3) The suggestion presupposed that every mechanism-phenomenon relationship is in fact deterministic.

4) There were counterexamples of (possibly) existing mechanism-phenomenon relationships that did not show the suggested kind of regularity. Most importantly, there are mechanism types whose instances usually do not bring about the phenomenon that the mechanism is supposed to explain, and there are phenomenon types whose instances are usually not brought about by instances of the mechanism that is supposed to explain the phenomenon.

In this section, I will introduce a new suggestion for how to understand regularity that avoids these problems. Roughly, the idea is the following: a mechanism type $M$ explains a phenomenon type $\mathrm{P}$ if and only if there is no other phenomenon type $\mathrm{P} *$ that is caused/constituted by more instances of $\mathrm{M}$ than $\mathrm{P}$; or if there is no other mechanism type $\mathrm{M}^{*}$ whose instances cause/constitute P more often than M. In the former case, the mechanismphenomenon sequence is what I will call 'comparatively regular'. In the latter case the sequence is 'comparatively reversely regular'. These two notions of regularity can be defined as follows:

(Comparative Regularity) A causal/constitutive sequence type consisting of type $\mathrm{A}$ and type B is comparatively regular iff there are more instances of A that cause/constitute an instance of B than instances of $\mathrm{A}$ that cause/constitute an instance of another type B*.

(Comparative Reverse Regularity) A causal/constitutive sequence type consisting of type $\mathrm{A}$ and type $\mathrm{B}$ is comparatively reversely regular iff there are more instances of B that are caused/constituted by an instance of 
A than instances of B that are brought about by an instance of another sequence type A*.

More specifically, my account of mechanistic type-level explanation is the following:

(Mechanistic Type-level Explanation) A mechanism type A explains a phenomenon type $\mathrm{B}$ iff the sequence type consisting of $\mathrm{A}$ and $\mathrm{B}$ is comparatively regular or comparatively reversely regular.

This idea is depicted in Figures 8 and 9.

[Insert Figure 8 here]

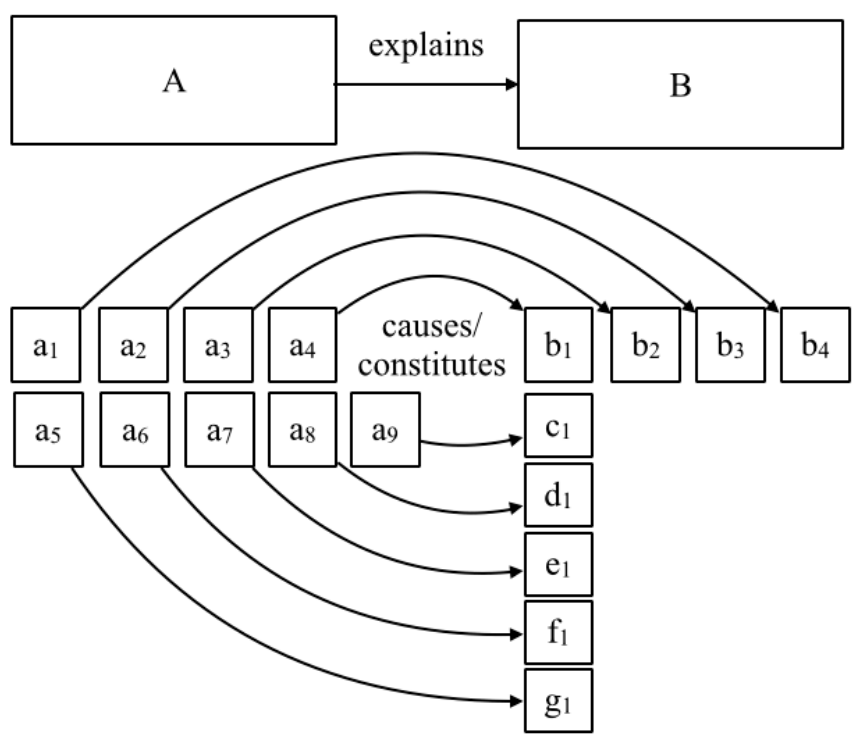

[Insert Figure 9 here]

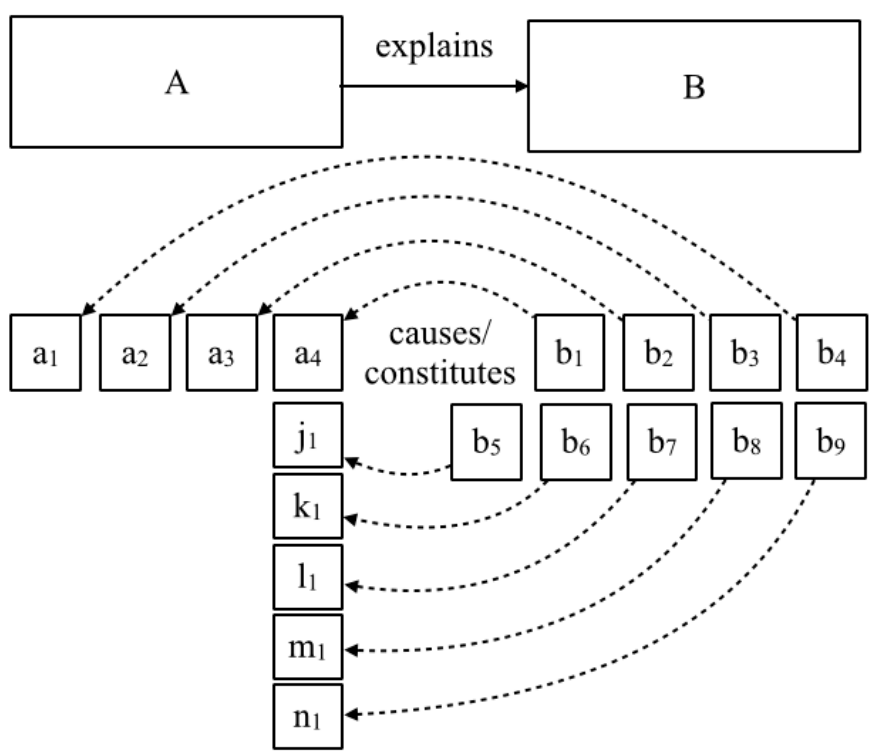


Take the neurotransmitter release mechanism again. This mechanism explains neurotransmitter release if and only if the effects it has in failure cases are not of the same type or if the alternative causes of neurotransmitter release do not constitute another mechanism type. Both conditions seem to be satisfied. Similarly, the cancer mechanism is comparatively reversely regular due to the fact that there are no alternative causes of cancer that form a unique mechanism type. But, interestingly, the mechanism-phenomenon relationship is not comparatively regular. The reason is that the alternative effects of the cancer mechanism (in failure cases) do form a unique phenomenon type. Bissell and Hines refer to the phenomenon type that occurs in failure cases as 'occult cancer' (Bissell and Hines [2011], p. 320).

The advantage of the present approach is that it solves the problems afflicting the previous suggestions (see beginning of this section). First, Comparative Regularity/Comparative Reverse Regularity does not require that we need a 'high degree' of regularity or Reverse Regularity in order to ground type-level explanations. The comparative notions of regularity and Reverse Regularity do not rely on an arbitrary determination of how many exceptions a particular mechanism-phenomenon sequence is allowed to have. Rather, how comparatively regular or reversely regular a specific mechanism-phenomenon sequence is depends on how regular or reversely regular it is compared to alternative mechanism-phenomenon sequences are-which is in principle an objective and definite issue. Second, the present account provides the resources to explain why mechanism-phenomenon relationships that instantiate Comparative Regularity and/or Comparative Reverse Regularity provide true type-level explanations. Roughly, if a mechanism-phenomenon relationship is comparatively regular, the mechanism grounds the explanation of the phenomenon because there is nothing else that the mechanism might explain better. If a mechanism-phenomenon relationship is comparatively reversely regular, the mechanism constitutes a true explanation of the phenomenon because there is nothing else that explains the phenomenon better. This provides a good way of describing how scientists might think about type-level explanations: they accept a type-level explanation as true if it can be excluded that there is a better explanation/another mechanism or causal sequence type that provides a better explanation (is more regular or reversely regular) than the mechanism at issue. Third, invoking the notions of Comparative Regularity and Comparative Reverse Regularity also avoids problem 3) since these notions allow for mechanism-phenomenon relationships to be non-deterministic. Fourth, based on these notions, we can accept that a mechanism-phenomenon relationship is regular even if the mechanism usually does not bring about the phenomenon, or the phenomenon is usually not 
brought about by the mechanism. I conclude that the comparative notions of regularity and Reverse Regularity provide a promising account of mechanistic type-level explanation.

\section{Multiple Realization, Multifunctionality and the Individuation of Types}

There is an obvious objection to the idea that the comparative notions of regularity and Reverse Regularity suffice for grounding type-level mechanistic explanations. My definition of mechanistic type-level explanation requires that there are no alternative phenomena that are more or equally likely to occur than the original phenomenon given the mechanism, or that there are no alternative mechanisms that are more or equally likely to occur than the original mechanism given the phenomenon. But surely there might be phenomena that can be due to more than one mechanism (see Section 4). Even more, my approach to mechanistic type-level explanation seems to exclude the possibility of multiple realization by definition (the possibility that there could be phenomena that can be due to more than one mechanism). Plausibly, cases of multiple realization might exist, and in these cases a phenomenon has more than one true mechanistic explanation.

(Multiple Realization) A phenomenon B might be multiply realized by two distinct mechanism types $\mathrm{A}$ and $\mathrm{J}$. In this case, $\mathrm{A}$ and $\mathrm{J}$ both explain $\mathrm{B}$.

We need independent criteria to be able to distinguish between cases of multiple realization and cases where a mechanism-phenomenon relation is simply not reversely regular. Plausibly, cases of multiple realization occur when a phenomenon exists in different contexts. In each context a different mechanism is responsible for the same phenomenon. An example of multiple realization might be the vision mechanism. Some instances of the vision mechanism involve ommatidia, some do not. Still, mechanisms involving ommatidia explain vision because they constitute one of various different types of vision mechanisms. The difference between these types is that they are instantiated in different animals, like those involving ommatidia are instantiated in insects (and, hence, they occur in different contexts).

The counterpart to multiple realization is what I will call 'multifunctionality'. Multifunctionality concerns the possibility of a mechanism bringing about more than one phenomenon.

(Multifunctionality) A mechanism A might bring about two distinct phenomena B and F. In this case, A explains B as well as F. 
Also, multifunctionality should not be excluded by definition, given that it constitutes a scenario that is accepted in the sciences. One example of multifunctionality might be the mechanism that is responsible for DNA-replication as well as re-replication:

the replication and re-replication mechanisms are the same, even though replication is said to be a normal process and re-replication an abnormal one [...] In both processes pre-RCs must be assembled, licensed and then fired and in all of these events the same proteins take part. For an abnormal process (rereplication) to occur an abnormal surrounding is necessary which is a result of impaired replication regulation [...]. (Mazurczyk and Rybaczek [2015], p. 31)

As before, we need independent criteria to be able to identify cases of multifunctionality. Plausibly, one should find differences in the contexts in which mechanism A brings about B and contexts where A brings about F. This idea is supported by the example where 'an abnormal surrounding is necessary' for re-replication to occur.

Surely, whether we are dealing with a case of multiple realization or multiple functionality or not crucially depends on how we individuate mechanism and phenomenon types. Two questions have to be answered:

1) When do two mechanism/phenomenon tokens belong to the same mechanism/phenomenon type?

2) How fine- or coarse-grained should we describe mechanism/phenomenon types?

The first question aims at determining which features of, for example, a mechanism are essential for its individuation. Consider two mechanisms that differ only in that one involves an entity and an activity the other does not involve. Are these two mechanisms of the same type? One answer might be that the two mechanisms are of the same type, if and only if they bring about the same phenomenon. The problem with this suggestion is that it does not allow for multiple realization. Intuitively, the identity of a mechanism type does not only depend on the phenomenon it produces but also on the entities and activities and their organization that make up the mechanism. Multiple realization, rather, should be taken to obtain if there are two or more mechanisms that 'do the same but in different ways' (Shapiro [2000]). Then, the question is, when mechanisms should be taken to do the same in different ways. Is the fact that one mechanism is composed of an acting entity that the other mechanism is not composed of sufficient to grant that the former works in a different way?

The second question asks for how to pick out types. Depending on which description is used, different things in the world are singled out. When referring to entities, one could, for example, use a description that picks out entities based on their functional features, another description picks out objects based on their structural features. Some descriptions are more 
abstract and rather general others describe many details. Type-level descriptions of mechanisms that abstract from various details are what MDC ([2000]) and Craver ([2006]) call 'mechanism schema'.

Scientists [...] are typically interested in types of mechanisms, not all the details needed to describe a specific instance of a mechanism. We introduce the term 'mechanism schema' for an abstract description of a type of mechanism. A mechanism schema is a truncated abstract description of a mechanism that can be filled with descriptions of known component parts and activities [...] Often, scientists use schema terms, such as 'transcription' and 'translation,' to capture compactly many aspects of the underlying mechanism. (MDC [2000], p. 16)

For example, describing a mechanism or a step in a mechanism as 'transcription' ignores the specific details of what exactly is going on in transcription. The corresponding type-level description is rather coarse-grained, and thus various concrete causal sequences might fall under this type. A more fine-grained type-level description of transcription might not include as many instances as the coarse-grained description.

A satisfying analysis of how to individuate mechanism and phenomenon types in the context of mechanistic explanation cannot be provided here. Surely, it is a crucial question not only with regard to the purposes of this paper but to the general account of mechanistic explanation. An account of mechanism and phenomenon types has to account for how scientists proceed in practice. Here, it might be fruitful to look at the methods of how scientists operationalize the phenomena they want to explain (operationalization might be seen as a process that starts with a rather general type description that is specified such that in the end individuals can be identified that can be investigated) (Feest [2005], [2010]; Sullivan [2009]). Plausibly, the identification of types in these contexts depends to a high degree on pragmatic considerations. In his discussion of the question whether mechanisms can ground natural kinds, Craver ([2009], p. 591) concludes that 'human perspectives and conventions enter into judgments about how mechanisms should be types and individuated'. Hence, it is not an objective matter of how mechanism tokens are subsumed under type descriptions. How you individuate mechanisms and phenomena depends on what you want to explain, what you know, what interests you, what is actually possible to investigate given your methods, tools and so on. One consequence of this would be that there is more than one way of dividing mechanisms and phenomena into types. This again has an interesting consequence with regard to type-level mechanistic explanation: whether a certain mechanism-phenomenon relation is comparatively regular and comparatively reversely regular, and thus, whether a particular mechanism explains a certain phenomenon depends to a big part on how you individuate the types, and therefore on pragmatic considerations. 


\section{Conclusion}

In order for a mechanistic explanation to be true it has to describe existing mechanisms. How can this idea be applied to type-level mechanistic explanation? What renders a mechanistic type-level explanation a true explanation? According to the account I developed in this paper, a mechanism type explains a particular phenomenon type if and only if the mechanismphenomenon sequence is comparatively regular or comparatively reversely regular. This account allows for stochastic mechanisms and even mechanisms that are rather unlikely to produce their phenomena to be truthmakers of type-level mechanistic explanation. Furthermore, based on this account, we can explain why regularity and Reverse Regularity ground mechanistic type-level explanation.

\section{Acknowledgments}

I am grateful to Marshal Abrams, Carl Craver, Alexander Dinges, Florian Fischer and Thomas Krödel for comments on earlier drafts of this paper. Furthermore, I presented the paper on several occasions where I got helpful comments: I thank the participants of the Workshop Realizability and the Levels of Reality in Paris 2014, the audience of my talk at the History \& Philosophy of Science \& Medicine Seminar Series at Washington University in St. Louis 2014, of the Current Issues in Philosophy lecture series at the School of Mind and Brain in Berlin 2014, at the Workshop Inference, Explanation \& Prediction in the Mind/Brain Sciences (here I especially thank Edouard Machery) at the School of Mind and Brain in Berlin 2015, the audience at the Nordic Network of the Philosophy of Science meeting in Helsinki 2015, the participants of Andreas Hüttemann's research colloquium in Cologne 2015, and Albert Newen and my colleagues at Ruhr-University Bochum for helpful discussions. Finally, I want to thank three anonymous referees for their helpful comments.

Ruhr-University Bochum Department of Philosophy II Universitätsstraße 150 D-44780 Bochum beate.krickel@rub.de

\section{References}

Abrams, M. [2012]: 'Mechanistic Probability', Synthese, 187, pp. 343-75.

Andersen, H. K. [2012]: 'The Case for Regularity in Mechanistic Causal Explanation', Synthese, 189, pp. 415-32. 
Barros, B. D. [2008]: 'Natural Selection as a Mechanism', Philosophy of Science, 75, pp. 306-22.

Baumgartner, M. and Gebharter, A. [2015]: 'Constitutive relevance, mutual manipulability, and fat-handedness', British Journal for the Philosophy of Science, doi: 10.1093/bjps/axv003.

Bechtel, W. [2008]: Mental Mechanisms: Philosophical Perspectives on Cognitive Neuroscience, London: Routledge.

Bechtel, W. and Abrahamsen, A. [2005]: 'Explanation. A Mechanist Alternative', Studies in History and Philosophy of Biological and Biomedical Sciences, 36, pp. 421-41.

Bechtel, W. and Richardson, R. C. [1993]: Discovering Complexity: Decomposition and Localization as Strategies in Scientific Research, Princeton: PUP. Reprinted: Bechtel, W. and Richardson, R. C. [2010]: Discovering Complexity: Decomposition and Localization as Strategies in Scientific Research, second edition, Cambridge, MA: MIT Press/Bradford Books.

Bissell, M. J. and Hines, W. C. [2011]: 'Why Don't we Get More Cancer? A Proposed Role of the Microenvironment in Restraining Cancer Progression', Nature Medicine, 17, pp. 320-9.

Bogen, J. [2004]: 'Analyzing Causality: The Opposite of Counterfactual is Factual', International Studies in the Philosophy of Science, 18, pp. 3-26.

Bogen, J. [2005]: 'Regularities and Causality; Generalizations and Causal Explanations', Studies in History and Philosophy of Biological and Biomedical Sciences, 36, pp. 397-420.

Branco, T. and Staras, K. [2009]: 'The Probability of Neurotransmitter Release: Variability and Feedback Control at Single Synapses', Nature Reviews Neuroscience, 10, pp. 373-83.

Cartwright, N. [1983]: How the Laws of Physics Lie, New York, NY: Oxford University Press.

Craver, C. F. [2006]: 'When Mechanistic Models Explain', Synthese, 153, pp. 355-76.

Craver, C. F. [2007a]: Explaining the Brain, New York, NY: Oxford University Press.

Craver, C. F. [2007b]: 'Constitutive Explanatory Relevance', Journal of Philosophical Research, 32, pp. 3-20.

Craver, C. F. [2009]: 'Mechanisms and Natural Kinds', Philosophical Psychology, 22, pp. 575-94.

Craver, C. F. [2014]: 'The Ontic Account of Scientific Explanation', in M. I. Kaiser, O. R. Scholz, D. Plenge and A. Hüttemann (eds), Explanation in the Special Sciences: The Case of Biology and History, Synthese Library 367, pp. 27-52.

Couch, M. B. [2011]: 'Mechanisms and Constitutive Relevance', Synthese, 183, pp. 375-88.

DesAutels, L. [2011]: 'Against Regular and Irregular Characterizations of Mechanisms', Philosophy of Science, 78, pp. 914-25.

DesAutels, L. [2015]: 'Toward a Propensity Interpretation of Stochastic Mechanism for the Life Sciences', Synthese, 192, pp. 2921-53.

Feest, U. [2005]: 'Operationism in Psychology: What the Debate is About, What the Debate Should be about', Journal of the History of the Behavioral Sciences, 41, pp. 13149.

Feest, U. [2010]: 'Concepts as Tools in the Experimental Generation of Knowledge in Cognitive Neuropsychology', Spontaneous Generations: A Journal for the History and Philosophy of Science, 4, pp. 173-90.

Glennan, S. [1996]: 'Mechanisms and the Nature of Causation', Erkenntnis, 44, pp. 49-71.

Glennan, S. [2010]: 'Ephemeral Mechanisms and Historical Explanation', Erkenntnis, 72, pp. 251-66. 
Glennan, S. [2011]: 'Singular and General Causal Relations: A Mechanist Perspective', in P. K. Illari, F. Russo and J. Williamson (eds), Causality in the Sciences, Oxford: Oxford University Press, pp. 789-817.

Glennan, S. [under review]: The New Mechanical Philosophy.

Harbecke, J. [2010]: 'Mechanistic Constitution in Neurobiological Explanations', International Studies in the Philosophy of Science, 24, pp. 267-85.

Hausman, D.M. [2005]: 'Causal Relata: Tokens, Types, or Variables?' Erkenntnis, 63, pp. $33-54$.

Hempel, C. G. [1965]: Aspects of Scientific Explanation, New York, NY: Free Press.

Illari, P. M. [2013]: 'Mechanistic Explanation: Integrating the Ontic and Epistemic', Erkenntnis, 78, pp. 237-55.

Illari, P. M. and Williamson, J. [2011]: 'Mechanisms are Real and Local', in P. K. Illari, F. Russo and J. Williamson (eds), Causality in the Sciences, Oxford: Oxford University Press, pp. 818-44.

Illari, P. M. and Williamson, J. [2012]: 'What is a Mechanism? Thinking About Mechanisms Across the Sciences', European Journal for Philosophy of Science, 2, pp. 119-35.

Kaiser, M. and Krickel, B. [2016]: 'The Metaphysics of Constitutive Mechanistic Phenomena', British Journal for the Philosophy of Science, doi: 10.1093/bjps/axv058.

Machamer, P., Darden, L. and Craver, C. F. (MDC) [2000]: 'Thinking About Mechanisms', Philosophy of Science, 67, pp. 1-25.

Machamer, P. [2004]: 'Activities and Causation: The Metaphysics and Epistemology of Mechanisms', International Studies in the Philosophy of Science, 18, pp. 27-39.

Mazurczyk, M. and Rybaczek, D. [2015]: 'Replication and Re-replication: Different Implications of the Same Mechanism', Biochimie, 108, pp. 25-32.

Navarro, S., Li, F. and Lampe, J. [2011]: 'Mechanisms of Action of Isothiocyanates in Cancer Chemoprevention: An Update', Food and Function, 2, pp. 579-86.

Nicholson, D. J. [2012]: 'The Concept of Mechanism in Biology', Studies in History and Philosophy of Biological and Biomedical Sciences, 43, pp. 655-64.

Railton, P. [1978]: 'A Deductive-Nomological Model of Probabilistic Explanation', Philosophy of Science, 45, pp. 206-26.

Romero, F. [2015]: 'Why There Isn't Inter-Level Causation in Mechanisms', Synthese, 192, pp. 3731-55.

Salmon, W. [1971]: 'Statistical Explanation', in W. Salmon (ed.), Statistical Explanation and Statistical Relevance, Pittsburgh: University of Pittsburgh Press, pp. 29-87.

Salmon, W. [1984]: 'Scientific Explanation: Three Basic Conceptions', PSA: Proceedings of the Biennial Meeting of the Philosophy of Science Association, Volume II: Symposia and Invited Papers, pp. 293-305.

Salmon, W. [1989]: Four Decades of Scientific Explanation, Pittsburgh: University of Pittsburgh Press.

Salmon, W. [1998]: Causality and Explanation, New York, NY: Oxford University Press.

Scriven, M. [1959]: 'Explanation and Prediction in Evolutionary Theory', Science, 30, pp. 477-82.

Shapiro, L. [2000]: 'Multiple Realizations', Journal of Philosophy, 97, pp. 635-54.

Sheredos, B. [2015]: 'Re-reconciling the Epistemic and Ontic Views of Explanation (Or, Why the Ontic View Cannot Support Norms of Generality)', Erkenntnis, doi: 10.1007/s10670-015-9775-5.

Sigurbjörnsdóttir, S., Mathew, R. and Leptin, M. [2014]: 'Molecular mechanisms of de novo lumen formation', Nature Reviews Molecular Cell Biology, 15, pp. 665-76.

Sullivan, J. [2009]: 'The Multiplicity of Experimental Protocols: A Challenge to Reductionist and Non-reductionist Models of the Unity of Neuroscience', Synthese, 176, pp. 511-39. 
Tucci, D. L. [2013]: 'Dizziness and Vertigo', Merck Manuals Professional Edition, $<$ www.merckmanuals.com/professional/ear-nose-and-throat-disorders/approachto-the-patient-with-ear-problems/dizziness-and-vertigo $>$.

Wright, C. D. [2012]: 'Mechanistic Explanation without the Ontic Conception', European Journal for Philosophy of Science, 2, pp. 375-94.

\section{Appendix: Figure Captions}

Figure 1: Diagram of the neurotransmitter release mechanism (my illustration).

Figure 2: A explains B iff every instance of A causes or constitutes an instance of B.

Figure 3: Stochastic mechanisms - not every instance of A causes/constitutes an instance of B.

Figure 4: High-failure mechanisms: most instances of A do not cause/constitute instances of B.

Figure 5: Diagram of the cancer mechanism (my illustration based on Navarro (2011)).

Figure 6: Reverse Regularity: A explains B if every instance of B is caused/constituted by an instance of A. (The doted arrows indicate that the b's are caused or constituted by the a's)

Figure 7: Some instances of B might not be caused or constituted by an instance of A. The dotted lines indicate the 'being caused/constituted by' relation.

Figure 8: Comparative regularity: A explains B if more as bring about bs than as bring about any other type (indicated by different letters c, d, e, f, g).

Figure 9: Comparative Reverse Regularity: A explains B if more bs are brought about by as than bs brought about by any other type (indicated by different letters j, k, 1, m, n). 